\title{
Treatment of multiple craniofacial osteomas by endoscopic approach
}

\author{
Hyokyung Yoo, \\ Gyeonghyeon Doh, \\ Baek Kyu Kim \\ Department of Plastic and \\ Reconstructive Surgery, Seoul National \\ University Bundang Hospital, Seoul \\ National University College of Medicine, \\ Seongnam, Korea
}

This article contains Supplemental Video S1.

\begin{abstract}
Osteomas are benign osteogenic neoplasms that usually occur as solitary craniofacial lesions. Multiple osteomas are rare, particularly those that do not occur as part of an associated syndrome. We report a case of a 72-year-old woman who presented with multiple bony protrusions over the forehead and scalp. She denied any diagnosis of syndromes that are known to be associated with osteomas. Surgical excision was performed by endoscopically resecting more than 30 osteomas of the frontal and bilateral parietal bones. Compared with conventional surgical excision through a direct incision, endoscopic-assisted surgery is a simple and effective method for the treatment of multiple craniofacial osteomas and is associated with excellent cosmetic outcomes and no neurovascular complications.
\end{abstract}

Keywords: Endoscopy / Gardner syndrome / Osteoma

\section{INTRODUCTION}

Osteomas are benign osteogenic neoplasms, which occur secondary to the proliferation of compact or cancellous bone and are observed in $0.01 \%$ to $0.43 \%$ of the general population. Based on the origin of the tumor, osteomas are classified into central, peripheral, and extra-skeletal types [1-3]. Although most peripheral osteomas show benign characteristics, osteomas that increase in size, particularly those at prominent sites such as the face, necessitate surgical intervention. Osteomas usually present as solitary lesions as few reports in the available literature have described multiple osteomas [4]. We report a rare case of non-syndromic multiple peripheral osteomas of the craniofacial bones in a patient who was surgically treated with endoscopic-assisted excision.

\footnotetext{
Correspondence: Baek Kyu Kim

Department of Plastic and Reconstructive Surgery, Seoul National University Bundang Hospital, Seoul National University College of Medicine, 82 Gumi-ro 173beon-gil, Bundang-gu, Seongnam 13620, Korea

E-mail: plasrecon@gmail.com

Received July 31, 2020 / Revised August 18, 2020 / Accepted August 19, 2020
}

\section{CASE REPORT}

A 72-year-old woman presented with a greater than 10-year history of multiple bony prominences of the forehead. Each mass measured approximately $1 \mathrm{~cm}$ in diameter, and palpation showed hard, immobile, non-tender, oval-shaped elevated lesions (Fig. 1A). The patient denied a history of trauma to the area, and no other cutaneous lesions were observed elsewhere on her body. She denied diagnosis of any syndromes or diseases, except for well-controlled chronic hypertension and diabetes. The endogastroduodenoscopy and colonoscopy performed to rule out Gardner's syndrome did not reveal any abnormalities of the gastrointestinal system. Intraoral evaluation also did not reveal any dental abnormalities, such as impacted or unerupted teeth. The preoperative computed tomography of craniofacial bones revealed multiple, small well-circumscribed lesions with density matching that of the surrounding osseous tissue, suggesting multiple peripheral osteomas of more than 30 lesions (Fig. 2A and B), primarily involving the frontal bone, as well as the upper aspects of the bilateral parietal bones.

Using the monitored anesthesia care protocol, we made two 

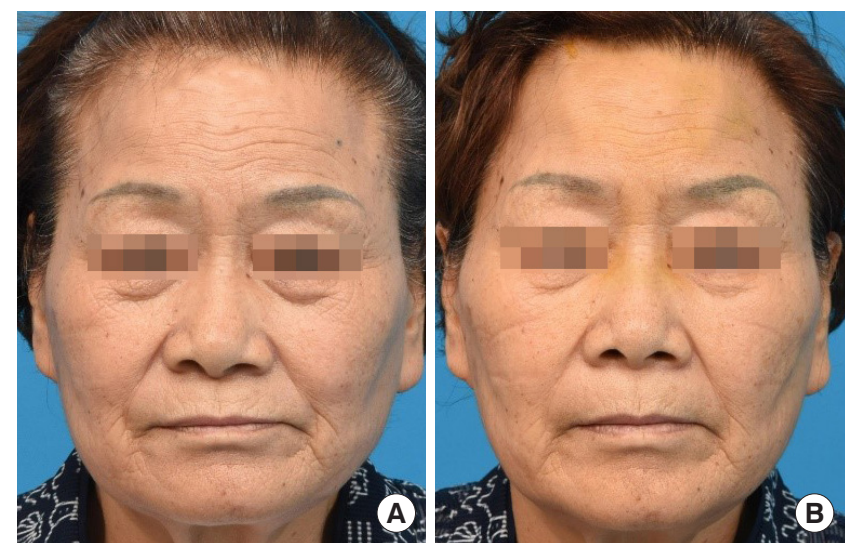

Fig. 1. (A) Preoperative photograph of a 72-year-old female with multiple osteomas in the forehead. (B) Postoperative photograph at 10 days after the surgical treatment.

small longitudinal incisions posterior to the hairline, and subperiosteal dissection was performed throughout the forehead via an endoscopic approach. Using osteotomes of varying degrees of curvature, we easily removed the well-defined masses, which were attached to the cortical bone, without destruction of the surrounding bone (Supplementary Video S1). The uneven surface was smoothened with a rasp to achieve a favorable contour of the forehead. The lesions posterior to the hairline that were cosmetically acceptable were left unremoved. A negative pressure drain was inserted through one of the incisions, and the wound was closed in layers.

The drain was removed on postoperative day 5 , and the sutures were removed on postoperative day 10 . We were able to observe aesthetically satisfactory outcomes without any postoperative complications, including hematoma, nerve injury, or any remnant palpable lumps. Histopathological examination confirmed mature lamellated bone fragments, consistent with osteomas. Photographs and computed tomography scans obtained 10 days postoperatively revealed a relatively well-smoothened surface of the forehead (Figs. 1B, 2C and D).

\section{DISCUSSION}

An osteoma is a relatively common type of osteogenic neoplasm of the craniofacial area, observed in $0.01 \%$ to $0.43 \%$ of the general population. These lesions develop secondary to the proliferation of compact or cancellous bone and are associated with multiple etiopathogenetic factors, including trauma, inflammation, developmental disorders, and genetic defects [3].

Osteomas usually occur as solitary lesions, and few cases of multiple osteomas have been reported in the previous literature [4]. Castelino et al. [5] described non-syndromic multiple gigantic osteomas involving the fronto-ethmoidal, mastoid, oc-
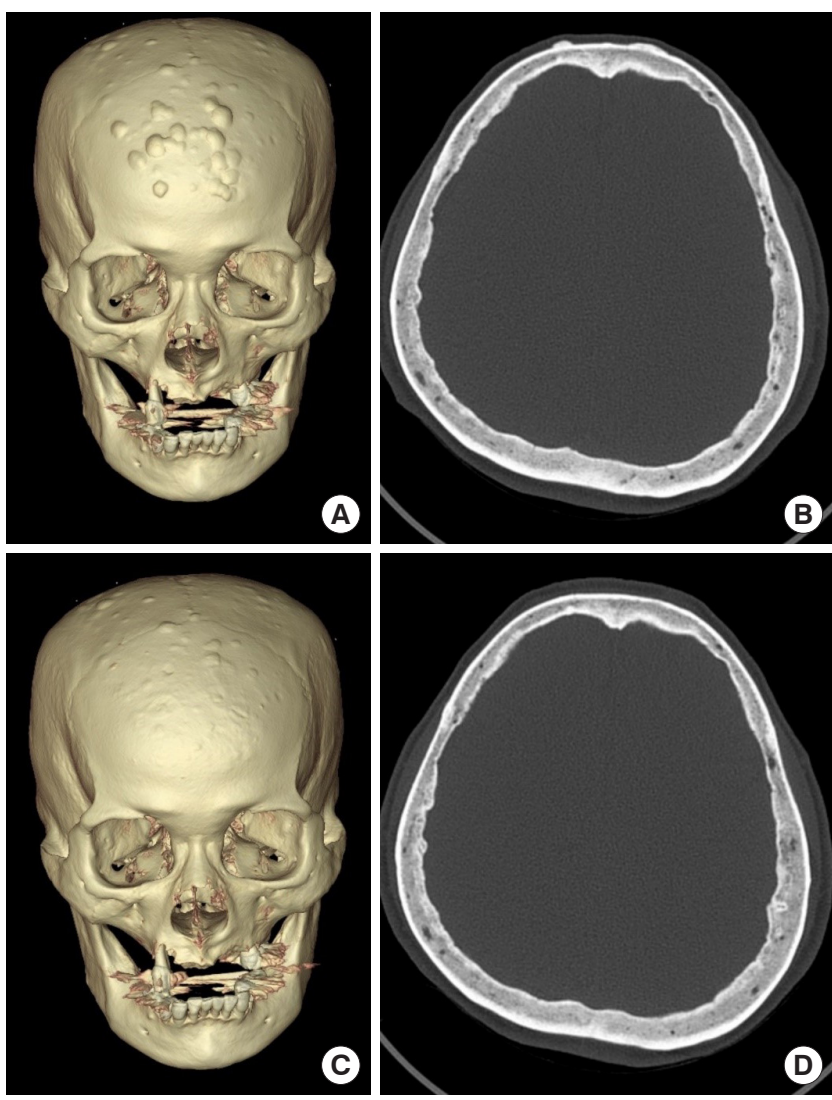

Fig. 2. (A, B) More than 30 lesions of osteoma in the frontal and bilateral parietal bones are shown in the preoperative computed tomography (CT) scan. (C, D) The multiple osteomas were all removed except for the ones behind the hairline in the postoperative CT scan.

cipital, mandibular, and maxillary areas in a 21-year-old woman, who was successfully treated with surgical resection. Shin et al. [6] also reported a case of non-syndromic multiple osteomas in a 54-year-old woman who presented with more than 160 osteomas involving the frontal, parietal, and occipital bones. Notably, in most patients, multiple lesions occur as a clinical manifestation of Gardner's syndrome, an inherited autosomal dominant condition that presents with colorectal polyposis, multiple osteomas, impacted or unerupted teeth, and cutaneous tumors. Bony lesions in patients with Gardner's syndrome usually include mandibular osteomas; Utsunomiya and Nakamura [7] reported that among 15 families with familial polyposis coli, 27 of 29 patients presented with radiopaque mandibular lesions.

Our patient underwent endoscopic evaluation of the gastrointestinal tract to rule out Gardner's syndrome; however, we observed no evidence of multiple polyposis. Gastrointestinal evaluation is essential for the assessment of multiple osteomas because non-syndromic multiple osteomas without underlying syndromes are rare and usually precede the presentation of colonic polyposis in Gardner's syndrome. Therefore, craniofacial 
lesions can be considered sensitive markers for the early diagnosis of this condition [8].

Osteomas are classified into central, peripheral, and extraskeletal lesions, based on the origin and site of involvement $[1,2]$. Although most peripheral lesions present without accompanying symptoms, osteomas that occur over prominent areas, such as the forehead, often necessitate surgical removal owing to aesthetically unpleasant disfigurement of the face. Conventionally, forehead osteoma has been removed through direct incisions or via the coronal approach; however, endoscopic-assisted surgery has gained widespread acceptance for its efficacy and low complication rates [9-11].

No report in the available literature describes endoscopic-assisted resection of multiple craniofacial osteomas. The patient described in this study did not experience any symptoms associated with the osteomas, and surgery was performed solely for cosmetic correction of the facial disfigurement. For the best aesthetic results, two longitudinal minimal incisions were made posterior to the hairline to prevent visible postoperative scars. Moreover, the risk of accidental injury to the neurovascular structures of the forehead was minimized by making the incisions in longitudinal direction instead of transverse direction and by performing safe subperiosteal dissection under direct visualization of the field by the guidance of endoscopic light source. We observed no lumps of the forehead, hematoma, or nerve injury postoperatively, and the patient was satisfied with the results.

In conclusion, we report a rare case of non-syndromic multiple peripheral osteomas involving the craniofacial bones. Endoscopic-assisted surgery is a simple and effective approach for multiple craniofacial osteomas, with excellent cosmetic outcomes and minimal neurovascular complications.

\section{NOTES}

\section{Conflict of interest}

No potential conflict of interest relevant to this article was reported.

\section{Ethical approval}

The study was approved by the Institutional Review Board of Seoul National University Bundang Hospital (IRB No. B-2008/631-101) and performed in accordance with the principles of the Declaration of Helsinki. Written informed consent was obtained.

\section{Patient consent}

The patient provided written informed consent for the publication and the use of her images.

\section{ORCID}

Hyokyung Yoo

https://orcid.org/0000-0002-9171-0379

Gyeonghyeon Doh

https://orcid.org/0000-0002-0318-8147

Baek Kyu Kim

https://orcid.org/0000-0001-9974-5165

\section{Supplementary material}

Supplementary Video S1. The well-defined bony masses were easily removed without destructing the surrounding bone by using various degrees of curved osteotomes with the aid of visualization by endoscopic approach through the two minimal incisions behind the hairline.

Supplemental data can be found at: https://doi.org/10.7181/ acfs.2020.00402.voo1.

\section{REFERENCES}

1. Patel TR, Borah GL. Frontal bone periosteal osteomas. Plast Reconstr Surg 2004;114:648-51.

2. Eshed V, Latimer B, Greenwald CM, Jellema LM, Rothschild BM, Wish-Baratz S, et al. Button osteoma: its etiology and pathophysiology. Am J Phys Anthropol 2002;118:217-30.

3. Haddad FS, Haddad GF, Zaatari G. Cranial osteomas: their classification and management: report on a giant osteoma and review of the literature. Surg Neurol 1997;48:143-7.

4. Larrea-Oyarbide N, Valmaseda-Castellon E, Berini-Aytes L, Gay-Escoda C. Osteomas of the craniofacial region: review of 106 cases. J Oral Pathol Med 2008;37:38-42.

5. Castelino RL, Subhas BG, Shishir RS, Kumuda Arvind Rao HT. Multiple craniofacial osteomas: an isolated case. Arch Orofac Sci 2011;6:32-6.

6. Shin JY, Roh SG, Lee NH, Yang KM. A case report in treatment of the frontal sinus osteoma using cranial bone graft. J Korean Soc Plast Reconstr Surg 2010;37:309-12.

7. Utsunomiya J, Nakamura T. The occult osteomatous changes in the mandible in patients with familial polyposis coli. $\mathrm{Br} \mathrm{J}$ Surg 1975;62:45-51.

8. Bilkay U, Erdem O, Ozek C, Helvaci E, Kilic K, Ertan Y, et al. Benign osteoma with Gardner syndrome: review of the literature and report of a case. J Craniofac Surg 2004;15:506-9.

9. Onishi K, Maruyama Y, Sawaizumi M. Endoscopic excision of forehead osteoma. J Craniofac Surg 1995;6:516-8.

10. Lai CH, Sun IF, Huang SH, Lai CS, Lin SD. Forehead osteoma excision by endoscopic approach. Ann Plast Surg 2008;61:533-6.

11. Mun GH, Jung ES, Lim SY, Hyon WS, Bang SI, Oh KS. Excision of forehead osteomas: experience with 12 patients with use of an endoscopic technique. J Craniofac Surg 2006;17:426-30. 